\title{
Étude de la profondeur \\ du sillon alvéolo-lingual chez l'édenté complet marocain
}

\section{Study of the depth of the groove in the lingual alveolar Moroccan totally edentulous}

\section{Résumé}

MOTS-CLEFS :
- Joint, langue,
empreinte, profo
alvéolo-lingual
KEYWORDS:
- Joint, tongue,
footprint, depth,
alveolar-lingual

$\operatorname{AOS} n^{\circ} 278-2016$
La région linguale est une région riche en éléments anatomiques et histologiques entrant en contact avec la prothèse complète mandibulaire. La mobilisation de ces éléments par différents mouvements fonctionnels peut entrainer l'instabilité de la prothèse si ceux-ci ne sont pas correctement appréhendés lors de l'empreinte secondaire.

Notre étude a visé la comparaison de la profondeur du sillon alvéolo-lingual dans chaque région de l'arcade lors des différents mouvements de la langue chez une population marocaine édentée complète.

L'étude a révélé que chaque partie de la région linguale est influencée par plusieurs mouvements en même temps. Ces mouvements peuvent légèrement changer d'un patient ò l'autre d'où l'intérêt de faire effectuer tous les mouvements de la langue par le patient lors de l'empreinte secondaire.
Abstract

Lingual region is a region rich in anatomical and histological parts coming into contact with the mandibular complete denture. The mobilization of these elements by various functional movements mary cause the instability of the prosthesis if they are not properly understood in the secondary footprint. Our study aimed to compare the depth of the furrow alveolar-lingual in each region of the arch during various movements of the tongue in a complete edentulous Moroccan population. The study found that every part of the lingual region is influenced by several movements simultaneously. These movements may slightly change from one patient to another hence the interest to perform all movements of the tongue by the patient during the secondary footprint.

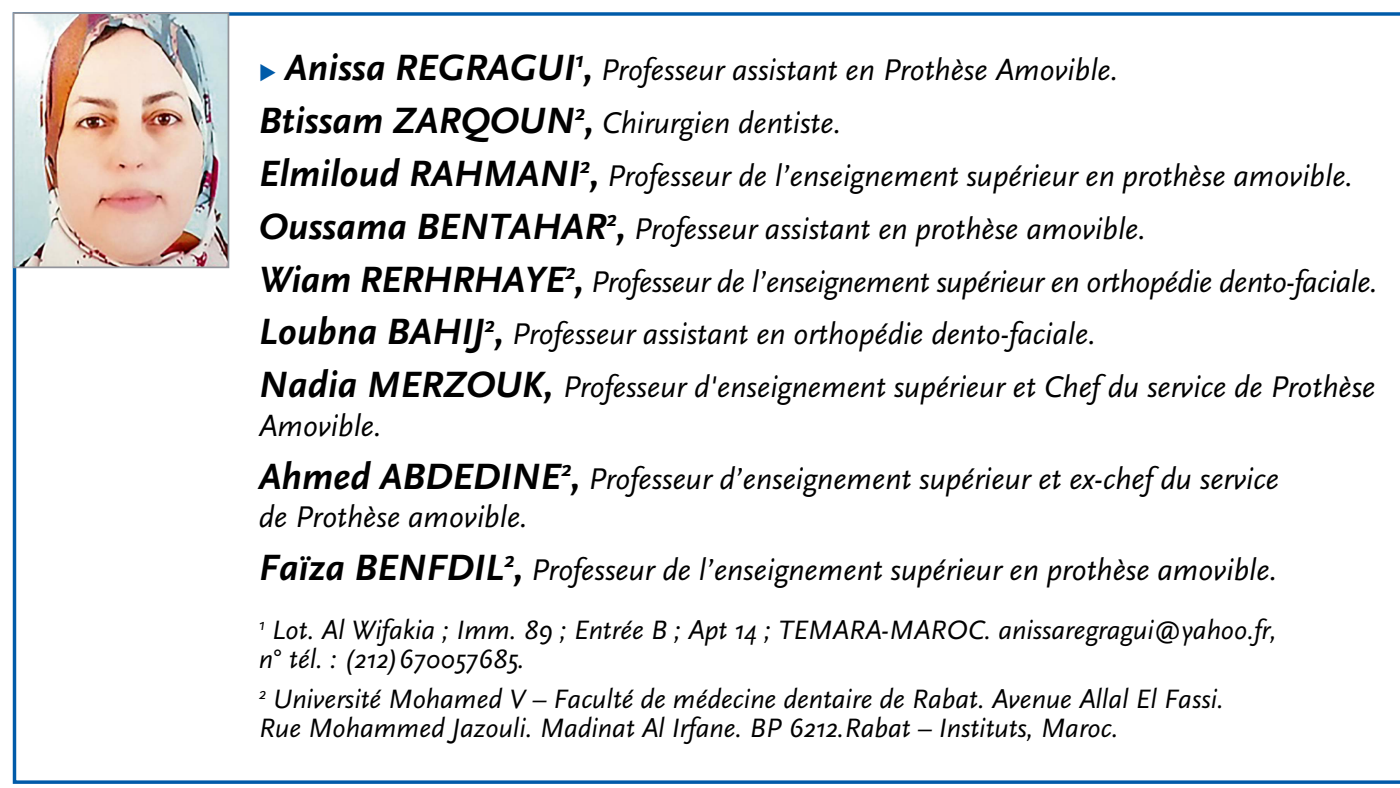




\section{INTRODUCTION}

La mandibule édentée présente toujours un certain défi pour le praticien. En effet, par rapport au maxillaire supérieur, à la surface d'appui prothétique réduite, s'ajoutent un jeu musculaire important et une muqueuse souvent fine recouvrant une crête résiduelle généralement irrégulière. [1]

L'enregistrement du joint périphérique au niveau lingual joue un rôle primordial dans la rétention de la prothèse mandibulaire. [2] [3] [4]

D’après Pompignoli M. [5], le joint sublingual est enregistré en demandant au patient de monter la langue au palais, de passer la langue d'une commissure à l'autre le long de la lèvre supérieure, de toucher l'intérieur de la joue gauche et droite, de prononcer " $\mathrm{Me}$ » « $\mathrm{Ma}$ » " Mi », de tirer la langue complètement en dehors et de déglutir.

Selon LEJOYEUX J. [6], la réalisation du joint sublingual se fait par section : la région mylohyoïdienne et rétromolaire droite et gauche.

Selon Jaudoin P., Millet C. et Mifsud S., [7] le joint lingual subit les actions du plancher de la bouche et surtout de la langue extrêmement mobile. L'image d'un joint enroulé de l'arrière vers l'avant et de l'intérieur à l'extérieur parait assez juste. [8] [9]

Devant cette divergence quant aux mouvements réalisés lors du remarginage, nous avons jugé nécessaire de réaliser une étude clinique permettant de mesurer les profondeurs du sillon alvéolo-lingual en fonction des différents mouvements réalisés.

\section{MATÉRIEL ET MÉTHODE}

Une étude descriptive et analytique a été menée pour évaluer la profondeur du sillon alvéolo-lingual lors de l'enregistrement du joint lingual chez les édentés complets et relever les mouvements linguaux influençant chaque région de l'arcade édentée mandibulaire tout en vérifiant l'influence de l'âge et du sexe sur la profondeur du sillon alvéolo-lingual.

L'étude s'est déroulée entre le 25-11-2010 et le 27-072011 au service de prothèse adjointe au centre de consultation et de traitement dentaire (CCTD) de Rabat. Un consentement écrit a été rédigé, traduit en langue arabe et mis à la disposition des patients après leur avoir expliqué le déroulement de l'étude mais la majorité des participants ont préféré nous donner leur accord verbal après explication de l'objectif et du déroulement de l'étude.

L'étude a porté sur un échantillon de 9 patients traités au centre de consultation et de traitement dentaire de Rabat et comportant 3 femmes et 6 hommes.
Les critères d'inclusion ont intéressé les patients édentés complets mandibulaires suivis au CCTD de Rabat entre le 25-11-2010 et le 27-07-2011 et acceptant de coopérer pour notre étude.

Les critères d'exclusion ont intéressé tout patient non coopérant, refusant de participer à cette étude, les patients parkinsoniens, les enfants en dessous de 18 ans et la présence de crête flottante ou de brides cicatricielles au niveau du fond du sillon alvéolo-lingual.

La première étape de l'étude a consisté à la réalisation d'un duplicata sur le modèle secondaire inférieur du patient, sur lequel nous avons confectionné un porte empreinte individuel (PEI) en résine autopolymérisable selon les limites tracées sur le nouveau modèle et qui se sont situées à $2 \mathrm{~mm}$ du fond du vestibule au niveau des bords et $3 \mathrm{~mm}$ au niveau des freins. Après, nous avons réalisé, en moufle, des duplicatas du PEI pour nous retrouver à la fin avec $8 \mathrm{PEI}$ identiques pour chaque patient.

La deuxième étape a consisté à l'enregistrement de tout le joint lingual en effectuant un seul mouvement pour chaque PEI. À cause de la rupture du silicone de moyenne viscosité (XANTOPREN ${ }^{\circledR}$ vert) durant la période de l'étude, et du coût excessif des polyéthers et des polyvinylsiloxanes pour le nombre de manipulations nécessaires, le matériau utilisé pour l'enregistrement du joint lingual dans notre étude a été l'oxyde zinc eugénol (l'impression past ${ }^{\circ}$ ) dont l'insertion en bouche a été légèrement retardée jusqu'à ce que sa consistance devienne adaptée pour l'enregistrement $\mathrm{du}$ joint périphérique. Tous les enregistrements ont été réalisés par le même opérateur.

Les mouvements de la lange demandés ont été : 1 - Lécher la lèvre supérieure, 2 - Lécher la lèvre inférieure, 3 - Langue à droite (dans la face interne de la joue), 4 - Langue à gauche (dans la face interne de la joue), 5 - Langue au palais, 6 - Déglutition, 7 - Protraction, 8 - Pousser le doigt du praticien.

Après l'obtention des modèles, nous avons procédé à l'enregistrement des mesures en divisant chaque modèle en 5 régions (Fig. 1) : la région rétro-molaire droite (Région $\mathrm{A})$, la région sublinguale postérieure droite (Région $\mathrm{B}$ ), la région sublinguale antérieure (Région $\mathrm{C}$ ), la région sublinguale postérieure gauche (Région $\mathrm{D})$, la région rétro-molaire gauche (Région $\mathrm{E}$ ). Chaque région a été divisée équitablement en trois petites régions. Les mesures ont été prises en distal, en mésial, et au milieu.

Nous avons obtenu à la fin, 31 mesures pour chaque modèle et 8 modèles pour chaque patient ce qui a donné 248 mesures pour chaque patient.

Les mesures ont été prises avec un pied à coulisse électronique pour chaque zone et ce du sommet de la crête au fond du sillon alvéolo-lingual. (Fig. 2) 


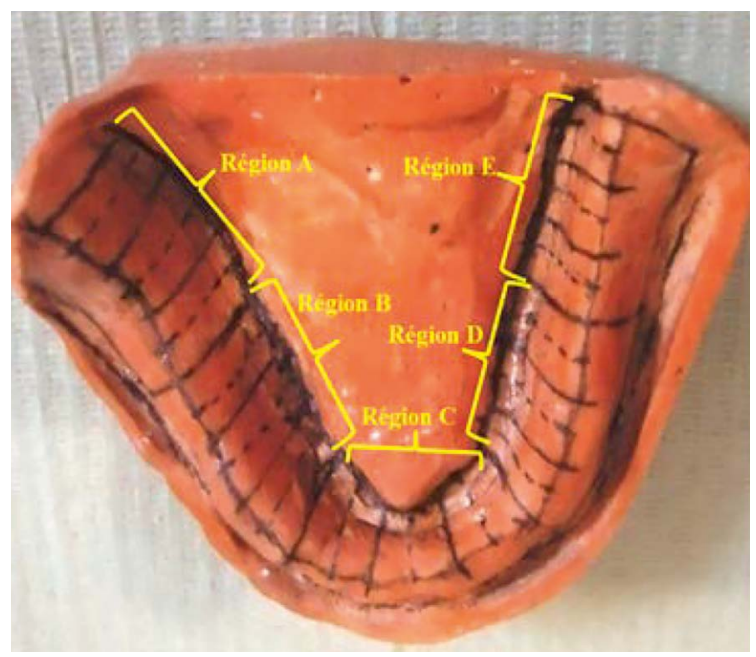

$\triangle$ Fig. 1 : Les 31 zones tracées sur le modèle.

\section{Analyse statistique}

La saisie des données a été réalisée dans le logiciel Excel 2007 et l'analyse par le logiciel SPSS 13.0 multilingue.

\section{RÉSULTATS}

Nous avons comparé, entre les 8 modèles, les profondeurs obtenues pour chaque zone. Nous avons choisi le mouvement fournissant la plus petite mesure parce que c'est le mouvement qui va le plus déstabiliser la prothèse et qui doit donc être enregistré lors de l'empreinte secondaire.

Nos résultats ont été résumés sous forme de cartes présentant la répartition des mouvements par région pour chaque patient puis pour tout l'échantillon (Fig. 3). Nous avons conclu que les mouvements obtenus différaient non seulement d'un patient à l'autre mais aussi à droite et à gauche dans la même arcade.

D'autre part, nous avons noté que le mouvement 6 (déglutition) a été absent au niveau des deux régions $\mathrm{A}$ et $\mathrm{E}$ et a présenté le pourcentage total le plus faible (4,3\%).

Les autres mouvements ont été tous présents avec une prédominance du mouvement 7 (protraction) dont le pourcentage total a été de $28,3 \%$. Il a été prédominant au niveau de toutes les régions (sauf la région $\mathrm{B}$ ) avec des pourcentages importants $(48,1 \%$ au niveau de la région $\mathrm{E})$ et ce d'une façon significative $(\mathrm{p}=0,004)$ (Tableau I).

La moyenne totale de la profondeur du sillon alvéolo-lingual a été de $7,70 \mathrm{~mm}$. La plus petite moyenne a été de 5,85, notée au niveau de la région C. La plus grande moyenne a été de 9,29 au niveau de la

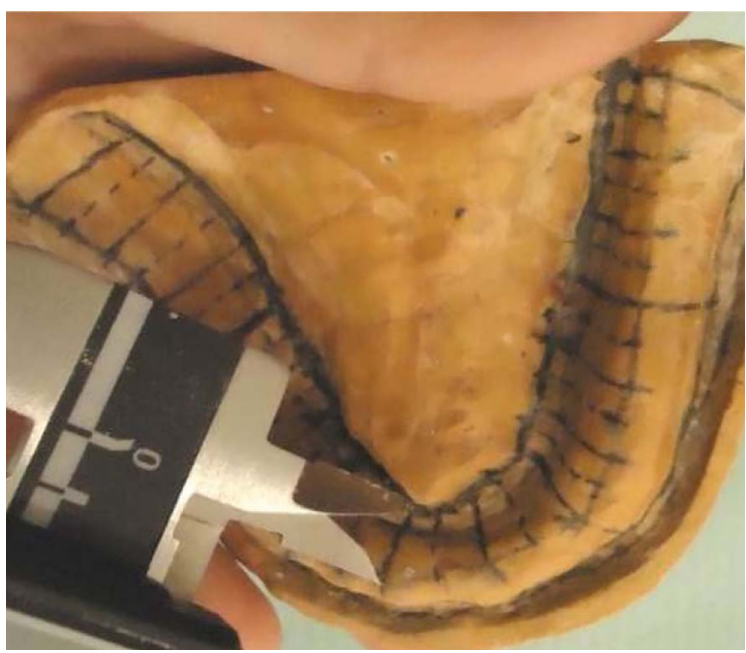

$\triangle$ Fig. 2 : La mesure prise avec un pied à coulisse électronique du sommet de la crête au fond du sillon alvéolo-lingual.

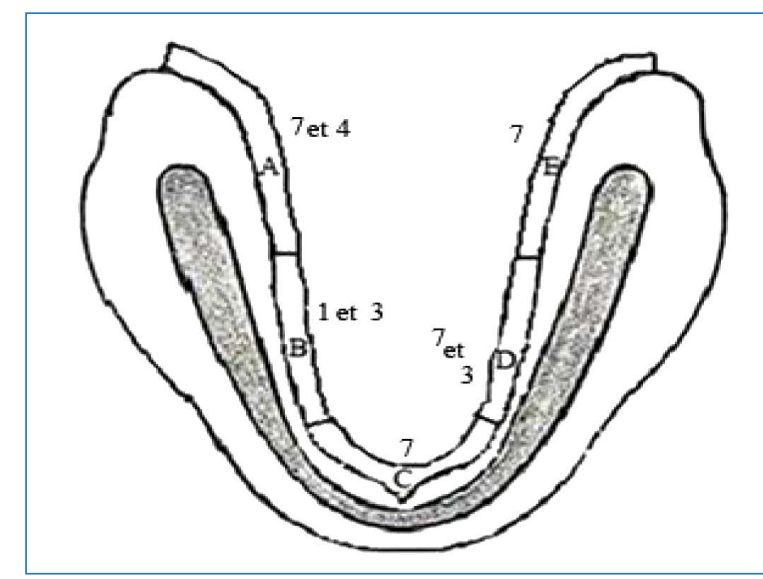

$\triangle$ Fig. 3 : Schéma montrant les mouvements prédominants pour chaque zone.

Le mouvement 1 : Lécher la lèvre supérieure.

Le mouvement 3 : Langue à droite (dans la face interne de la joue) Le mouvement $4:$ Langue à gauche (dans la face interne de la joue) Le mouvement 7 : Protraction de la langue.

région $\mathrm{A}$, suivie de la région $\mathrm{E}$ avec une moyenne de 9,24 (Tableau II et Fig. 4).

Pour la première tranche d'âge (moins de 50 ans), la plus petite moyenne a été enregistrée au niveau de la région $C$ avec une valeur de 6,52 .

Pour la deuxième tranche d'âge (de 51 à 60 ans), cette moyenne a été de 5,44 et elle a été notée également au niveau de la région $C$.

Pour la troisième tranche d'âge (plus de 61 ans), la plus petite moyenne a été enregistrée au niveau de la région $B$ avec une valeur de 6,24 suivie d'une valeur de 6,30 au niveau de la région C (Fig. 5).

La comparaison entre la moyenne totale des trois tranches d'âge a montré que la $1^{\text {re }}$ tranche d'âge présentait la valeur la plus élevée alors que la $2^{\mathrm{e}}$ tranche d'âge présentait la plus petite moyenne. La $3^{\mathrm{e}}$ tranche d'âge présentait une moyenne entre les deux autres (Tableau III). 
Tableau I : Répartition des mouvements selon les régions pour tout l'échantillon.

\begin{tabular}{|c|c|c|c|c|c|c|}
\hline $\mathrm{P}$ & \multicolumn{6}{|c|}{ Effectif (pourcentage) } \\
\hline Mouvements & $\mathbf{A}$ & B & C & D & $\mathbf{E}$ & Total \\
\hline $1:$ Lécher la lèvre supérieure & $11(17,5 \%)$ & $13(24,1 \%)$ & $7(13 \%)$ & $6(11,1 \%)$ & $1(1,9 \%)$ & $38(13,6 \%)$ \\
\hline $2:$ Lécher la lèvre inférieure & $7(11,1 \%)$ & $7(13 \%)$ & $9(16,7 \%)$ & $2(3,7 \%)$ & $8(14,8 \%)$ & $33(11,8 \%)$ \\
\hline 3 : Langue à droite & $2(3,2 \%)$ & $10(18,5 \%)$ & $9(16,7 \%)$ & $11(20,4 \%)$ & $9(16,7 \%)$ & $41(14,7 \%)$ \\
\hline 4: Langue à gauche & $14(22,2 \%)$ & $5(9,3 \%)$ & $4(7,4 \%)$ & $1(1,9 \%)$ & $0(0 \%)$ & $24(8,6 \%)$ \\
\hline 5: Langue au palais & $4(6,3 \%)$ & $6(11,1 \%)$ & $4(7,4 \%)$ & $10(18,5 \%)$ & $1(1,9 \%)$ & $25(9 \%)$ \\
\hline 6 : Déglutition & $0(0 \%)$ & $1(1,9 \%)$ & $7(13 \%)$ & $4(7,4 \%)$ & $0(0 \%)$ & $12(4,3 \%)$ \\
\hline $7:$ Protraction & $16(25,4 \%)$ & $7(13 \%)$ & $12(22,2 \%)$ & $18(33,3 \%)$ & $26(48,1 \%)$ & $79(28,3 \%)$ \\
\hline $8:$ Pousser le doigt du praticien & $9(14,3 \%)$ & $5(9,3 \%)$ & $2(3,7 \%)$ & $2(3,7 \%)$ & $9(16,7 \%)$ & $27(9,7 \%)$ \\
\hline Total & $63(100 \%)$ & $54(100 \%)$ & $54(100 \%)$ & $54(100 \%)$ & $54(100 \%)$ & $279(100 \%)$ \\
\hline $\mathrm{p}$ & \multicolumn{6}{|c|}{0,004} \\
\hline
\end{tabular}

Tableau II : Moyenne des profondeurs du sillon alvéolo-lingual ( $\mathrm{mm})$.

\begin{tabular}{|c|c|c|c|}
\hline Régions & Moyenne \pm écart type & Minimum & Maximum \\
\hline A & $9,29 \pm 2,79$ & 4,30 & 19,7 \\
\hline B & $6,83 \pm 2,2$ & 1,95 & 13,14 \\
\hline C & $5,85 \pm 1,77$ & 2,15 & 10,73 \\
\hline D & $7,05 \pm 1,68$ & 3,07 & 12,38 \\
\hline E & $9,24 \pm 2,55$ & 3,85 & 20,66 \\
\hline Total & $7,70 \pm 2,65$ & 1,95 & 20,66 \\
\hline
\end{tabular}

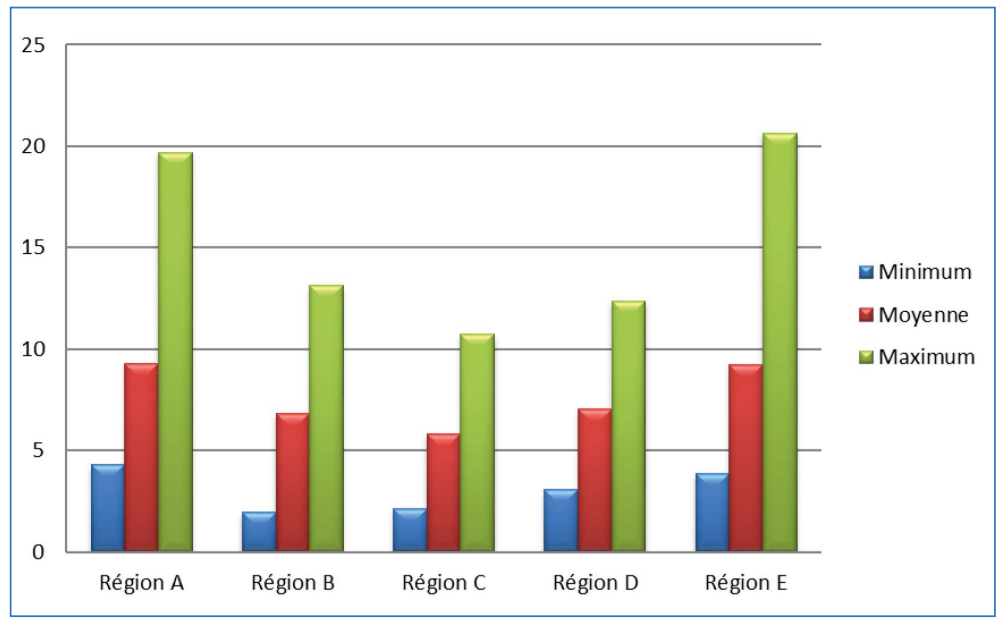

Fig. 4 : Moyenne des profondeurs du sillon alvéolo-lingual.

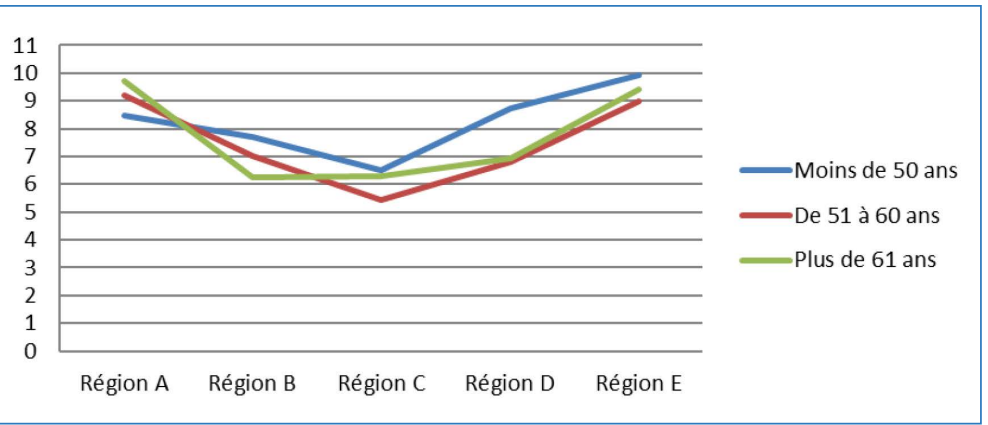

Fig. 5 : Comparaison des moyennes par tranches d'âge (mm).
La plus petite moyenne a été notée au niveau de la région $\mathrm{C}$ pour les deux sexes avec des valeurs de 5,71 et 6,12. Les profondeurs chez les hommes ont été supérieures aux profondeurs chez les femmes au niveau des 3 régions : région $\mathrm{A}$, région $\mathrm{D}$, région $\mathrm{E}$.

La différence entre les moyennes est significative entre les hommes et les femmes ( $p=0,05)$ (Tableau IV).

\section{DISCUSSION}

Pour remplir son rôle, la prothèse mandibulaire doit se situer dans une zone neutre où l'action musculaire doit être équilibrée et contre balancée par la force de rétention de la prothèse, d'où la nécessité d'un joint périphérique efficace [10] [11] [12].

Notre étude a montré que la région antérieure est influencée en premier lieu par le mouvement de protraction $(22,2 \%)$, suivi de tous les autres mouvements enregistrés. Ces mouvements, selon leur importance, sont : lécher la lèvre inférieure (16,7\%), langue à droite $(16,7 \%)$, lécher la lèvre supérieure $(13 \%)$, déglutition $(13 \%)$, langue à gauche $(7,4 \%)$, langue au palais $(7,4 \%)$, pousser le doigt du praticien $(3,7 \%)$.

Les résultats obtenus paraissent assez intéressants puisqu'ils concordent par :

$\checkmark$ le mouvement de "protraction", avec Pompignoli qui le considère parmi les mouvements importants à enregistrer dans cette région, contrairement aux autres auteurs.

I Le mouvement "lécher la lèvre inférieure", avec deux auteurs; Lejoyeux et E. Budtz-Jorgensen et R. Clavel. Ces auteurs réalisent ces mouvements avec les mouvements «lécher la lèvre supérieure ", retrouvé également dans nos résultats. Pompignoli réalise le mouvement « lécher la lèvre supérieure » 
Tableau III : Comparaison des moyennes par tranches d'âge (mm).

\begin{tabular}{|c|c|c|c|c|c|c|c|c|c|}
\hline \multirow{2}{*}{$\begin{array}{r}\text { Tranches d'âge } \\
\text { Mesure }\end{array}$} & \multicolumn{3}{|c|}{ Moins de 50 ans } & \multicolumn{3}{|c|}{ De 51 à 60 ans } & \multicolumn{3}{|c|}{ Plus de 61 ans } \\
\hline & $\begin{array}{l}\text { Moyenne } \\
\pm \text { écart } \\
\text { type }\end{array}$ & $\begin{array}{l}\text { Mini- } \\
\text { mum }\end{array}$ & $\begin{array}{l}\text { Maxi- } \\
\text { mum }\end{array}$ & $\begin{array}{l}\text { Moyenne } \\
\text { 土écart } \\
\text { type }\end{array}$ & $\begin{array}{l}\text { Mini- } \\
\text { mum }\end{array}$ & $\begin{array}{l}\text { Maxi- } \\
\text { mum }\end{array}$ & $\begin{array}{c}\text { Moyenne } \\
\text { 土écart } \\
\text { type }\end{array}$ & $\begin{array}{l}\text { Mini- } \\
\text { mum }\end{array}$ & $\begin{array}{l}\text { Maxi- } \\
\text { mum }\end{array}$ \\
\hline A & $8,49 \pm 1,07$ & 6,09 & 11,38 & $9,18 \pm 2,66$ & 4,30 & 17,18 & $9,73 \pm 3,29$ & 5,50 & 19,17 \\
\hline B & $7,70 \pm 0,54$ & 6,54 & 8,59 & $7,01 \pm 2,41$ & 2,20 & 13,14 & $6,24 \pm 2,06$ & 1,95 & 9,99 \\
\hline $\mathrm{C}$ & $6,52 \pm 1,03$ & 4,92 & 9,02 & $5,44 \pm 1,47$ & 2,17 & 9,95 & $6,30 \pm 2,2$ & 2,15 & 10,73 \\
\hline $\mathrm{D}$ & $8,71 \pm 1,06$ & 7,32 & 11,35 & $6,79 \pm 1,7$ & 3,07 & 12,38 & $6,93 \pm 1,52$ & 3,13 & 10,27 \\
\hline $\mathrm{E}$ & $9,91 \pm 1,74$ & 5,65 & 12,91 & $8,99 \pm 2,09$ & 3,85 & 15,05 & $9,42 \pm 3,32$ & 3,87 & 20,66 \\
\hline Total & $8,27 \pm 1,59$ & 4,92 & 12,91 & $7,54 \pm 2,56$ & 2,17 & 17,18 & $7,79 \pm 3,02$ & 1,95 & 20,66 \\
\hline
\end{tabular}

Tableau IV : Comparaison des moyennes selon le sexe ( $\mathrm{mm})$.

\begin{tabular}{|c|c|c|c|c|c|c|}
\hline Sexe & \multicolumn{3}{|c|}{ Femmes } & \multicolumn{3}{|c|}{ Hommes } \\
\hline Régions & $\begin{array}{l}\text { Moyenne } \\
\pm \text { écart } \\
\text { type }\end{array}$ & Minimum & Maximum & $\begin{array}{l}\text { Moyenne } \\
\pm \text { écart } \\
\text { type }\end{array}$ & Minimum & Maximum \\
\hline A & $8,22 \pm 1,8$ & 4,30 & 13,98 & $9,83 \pm 3,04$ & 4,41 & 19,17 \\
\hline B & $6,93 \pm 1,18$ & 4,33 & 10,27 & $6,78 \pm 2,57$ & 1,95 & 13,14 \\
\hline $\mathrm{C}$ & $6,12 \pm 2,06$ & 2,17 & 10,73 & $5,71 \pm 1,59$ & 2,15 & 9,95 \\
\hline $\mathrm{D}$ & $6,62 \pm 1,56$ & 3,07 & 10,27 & $7,26 \pm 1,71$ & 3,13 & 12,38 \\
\hline $\mathrm{E}$ & $8,75 \pm 1,92$ & 3,85 & 15,05 & $9,48 \pm 2,79$ & 3,87 & 20,66 \\
\hline Total & $7,36 \pm 1,99$ & 2,17 & 15,05 & $7,88 \pm 2,91$ & 1,95 & 20,66 \\
\hline $\mathrm{p}$ & \multicolumn{6}{|c|}{0,05} \\
\hline
\end{tabular}

indépendamment du mouvement «lécher la lèvre inférieure » contrairement aux autres auteurs.

\Le mouvement «déglutition », avec Pompignoli et E. Budtz-Jorgensen et R. Clavel.

I Les mouvements « langue au palais» avec les trois auteurs.

\ Le mouvement « langue à droite » « langue à gauche » avec Pompignoli.

IEt avec Mifsud par le mouvement «pousser le doigt».

Selon notre étude, tous les mouvements affectent, d'une façon ou d'une autre la stabilité de la prothèse au niveau de cette région, donc ils doivent tous être enregistrés, tout en insistant sur la protraction qui semble être le mouvement le plus déstabilisateur.

D'autre part, nous avons divisé la région postérieure dans notre étude en deux régions : régions moyennes (droite et gauche) et régions postérieures (droite et gauche).

\La région moyenne gauche : influencée par le mouvement de protraction puis viennent les autres mouvements, qui selon leur importance sont : la langue à droite $20,4 \%$, la langue au palais $18,5 \%$, lécher la lèvre supérieure $11,1 \%$, déglutition $7,4 \%$, lécher la lèvre inférieure $3,7 \%$, pousser le doigt $3,7 \%$, la langue à gauche $1,9 \%$.
La région moyenne droite : mobilisée surtout quand le patient lèche sa lèvre

supérieure. Là encore nous retrouvons les autres mouvements de la langue mais avec un ordre différent: la langue à droite $18,5 \%$, la protraction $13 \%$, lécher la lèvre inférieure $13 \%$, la langue au palais $11,1 \%$, la langue à gauche $9,3 \%$, pousser le doigt 9,3\%, la déglutition $1,9 \%$.

$\checkmark$ Les régions postérieures droite et gauche sont influencées de façon primordiale par la protraction, avec la présence des autres mouvements qui, encore une fois, ne figurent pas dans le même ordre à droite et à gauche.

\begin{tabular}{|c|c|}
\hline Région postérieure droite & Région postérieure gauche \\
\hline La langue à gauche $22,2 \%$ & La langue à droite $16,7 \%$ \\
\hline Lécher la lèvre supérieure $17,5 \%$ & Pousser le doigt $16,7 \%$ \\
\hline Pousser le doigt $14,3 \%$ & Lécher la lèvre inférieure $14,8 \%$ \\
\hline Lécher la lèvre inférieure $11,1 \%$ & Lécher la lèvre supérieure $1,9 \%$ \\
\hline La langue au palais $6,3 \%$ & La langue au palais $1,9 \%$ \\
\hline La langue à droite $3,2 \%$ & \\
\hline
\end{tabular}

En revenant à la littérature nous trouvons que certains auteurs divisent également cette région en deux régions et chacune est enregistrée par un certain nombre de mouvements. 
Selon E. Budtz-Jorgensen et R. Clavel [13], la région postérieure comporte :

I La zone sublinguale postérieure, et pour l'enregistrer on demande au patient d'effectuer les mêmes mouvements que pour la zone sublinguale antérieure: passer la langue sur la lèvre supérieure et inférieure, déglutir, lever la langue au palais.

\ La zone de la ligne oblique interne, qu'on enregistre par le mouvement de latéralité de la langue tout en touchant la face interne de la joue.

P. Jaudoin, C. Millet et S. Mifsud [7] divisent également la région postérieure en deux régions :
I Zone intermédiaire qu'on enregistre en demandant au patient de toucher la face interne de la joue d'un côté à l'autre.

$\checkmark$ Zone rétromolaire enregistrée en tirant la langue vers la commissure du côté opposé.

Les mouvements réalisés pour enregistrer les régions postérieures sont les mêmes pour le côté droit et le côté gauche.

Lejoyeux [6] enregistre les régions mylohyoïdienne et rétromolaires en demandant au patient d'effectuer les mêmes mouvements pour les deux régions et qui sont : protraction de la langue, déglutition,

Tableau V : Schémas récapitulant la répartition des mouvements par région.

\begin{tabular}{|c|c|c|}
\hline Auteur & Répartition & Mouvements \\
\hline \multirow[t]{2}{*}{$\begin{array}{l}\text { E. Budtz Jorgensen } \\
\text { et R. Clavel }\end{array}$} & $\begin{array}{l}\text { Zones sublinguales } \\
\text { postérieures et antérieures } \\
\text { et le frein lingual }\end{array}$ & $\begin{array}{l}\text { Passer la langue sur la lèvre supérieure et inférieure d'une commissure à } \\
\text { l'autre. } \\
\text { Déglutition. } \\
\text { Langue au palais. }\end{array}$ \\
\hline & $\begin{array}{l}\text { Zone de la ligne oblique } \\
\text { interne }\end{array}$ & $\begin{array}{l}\text { Toucher la face interne de la joue avec la pointe de la langue en passant par } \\
\text { dessus du PEI. }\end{array}$ \\
\hline Pompignoli & Joint sublingual & $\begin{array}{l}\text { Langue au palais. } \\
\text { Passer la langue d'une commissure à l'autre le long de la lèvre supérieure. } \\
\text { Toucher l'intérieure de la joue gauche et droite. } \\
\text { Prononcer Me Ma Mi. } \\
\text { Protraction. } \\
\text { Déglutition. }\end{array}$ \\
\hline \multirow{2}{*}{ Joseph Lejoyeux } & $\begin{array}{l}\text { Région mylohyoïdienne } \\
\text { et rétromolaire }\end{array}$ & $\begin{array}{l}\text { Protraction de la langue. } \\
\text { Déplacement de la pointe vers la droite et vers la gauche. } \\
\text { Déglutition. }\end{array}$ \\
\hline & Joint sublingual & $\begin{array}{l}\text { Reculer la langue. } \\
\text { Lever la pointe. } \\
\text { Lécher la lèvre supérieure puis inférieure. }\end{array}$ \\
\hline \multirow{3}{*}{$\begin{array}{l}\text { P. Jaudoin, C. Millet } \\
\text { et S. Mifsud }\end{array}$} & Zone rétromolaire. & Tirer la langue obliquement vers la commissure opposée. \\
\hline & Zone intermédiaire. & Toucher la face interne de la joue avec la langue (de côté à l'autre). \\
\hline & Zone antérieure. & $\begin{array}{l}\text { Demander au patient de pousser avec la langue sur un doigt en simulant une } \\
\text { apparence de baiser. }\end{array}$ \\
\hline \multirow{5}{*}{ Notre étude } & Région postérieure droite. & $\begin{array}{l}\text { Protraction }(25,4 \%) \text {, langue à gauche }(22,2 \%) \text {, lécher la lèvre supérieure } \\
(17,5 \%) \text {, pousser le doigt }(14,3 \%), \text { lécher la lèvre inférieure }(11,1 \%) \text {, langue } \\
\text { au palais }(6,3 \%) \text {, langue à droite }(3,2 \%) \text {. }\end{array}$ \\
\hline & Région postérieure gauche. & $\begin{array}{l}\text { Protraction }(48,1 \%) \text {, langue à droite }(16,7 \%) \text {, pousser le doigt }(16,7 \%) \text {, } \\
\text { lécher la lèvre inférieure }(14,8 \%) \text {, lécher la lèvre supérieure }(1,9 \%) \text {, langue } \\
\text { au palais }(1,9 \%) .\end{array}$ \\
\hline & Région moyenne droite. & $\begin{array}{l}\text { Lécher la lèvre supérieure }(24,1 \%) \text {, langue à droite }(18,5 \%) \text {, protraction } \\
(13 \%) \text {, lécher la lèvre inférieure }(13 \%) \text {, langue au palais }(11,1 \%) \text {, langue à } \\
\text { gauche }(9,3 \%) \text {, pousser le doigt }(9,3 \%) \text {, déglutition }(1,9 \%) \text {. }\end{array}$ \\
\hline & Région moyenne gauche. & $\begin{array}{l}\text { Protraction }(33,3 \%) \text {, langue à droite }(20,4 \%) \text {, langue au palais }(18,5 \%) \text {, } \\
\text { lécher la lèvre supérieure }(11,1 \%) \text {, déglutition }(7,4 \%) \text {, lécher la lèvre } \\
\text { inférieure }(3,7 \%) \text {, pousser le doigt }(3,7 \%) \text {, langue à gauche }(1,9 \%) \text {. }\end{array}$ \\
\hline & Région antérieure. & $\begin{array}{l}\text { Protraction }(22,2 \%) \text {, lécher la lèvre inférieure }(16,7 \%) \text {, langue à droite } \\
(16,7 \%) \text {, déglutition }(13 \%) \text {, lécher la lèvre supérieure }(13 \%) \text {, langue à } \\
\text { gauche }(7,4 \%) \text {, langue au palais }(7,4 \%) \text {, pousser le doigt }(3,7 \%) \text {. }\end{array}$ \\
\hline
\end{tabular}

NB : les mouvements concernant notre étude sont cités par ordre décroissant de leurs pourcentages. 
déplacement de la pointe vers la droite et vers la gauche.

Le mouvement de latéralité de la langue semble aussi avoir une importance particulière chez ces auteurs au niveau des régions postérieures. Chose que nous avons trouvée également dans notre étude vu que ce mouvement est classé deuxième au niveau de toutes les régions.

Pour récapituler nous présentons le tableau $\mathrm{V}$ ci-contre.

Quand nous comparons les résultats obtenus à

droite et à gauche, nous observons que les mouvements relevés à droite ne figurent pas dans le même ordre d'importance que les mouvements relevés à gauche. Cela peut être dû aux profondeurs du sillon alvéolo-lingual qui ne sont pas les mêmes à droite et à gauche chez le même sujet. Cette asymétrie pourrait être expliquée par l'asymétrie de l'arcade édentée mandibulaire et aussi par l'histoire dentaire de chaque patient (type d'extraction, interventions chirurgicales, tics et habitudes, durée de l'édentement). [14] [15]

Nous pourrions aussi incriminer les fonctions de la langue et qui sont en rapport avec les habitudes du patient comme la mastication unilatérale, qui fait que le patient a tendance à mobiliser sa langue beaucoup plus vers un côté que vers l'autre. La coopération du patient joue un rôle important dans la réalisation d'un bon enregistrement. Au cours de notre étude, nous avons rencontré des patients qui, au début de la séance de l'enregistrement, n'étaient pas aussi à l'aise qu'à la fin de la séance.

La profondeur du sillon alvéolo-lingual va en décroissant d'arrière en avant. Ceci est compréhensible si nous considérons le travail de SCHREINEMAKERS, qui affirme que la plus grande intensité des forces musculaires se manifeste, au niveau du plancher buccal, toujours au centre de la face linguale et diminue progressivement du centre vers la face latérale, pour aboutir à une valeur nulle dans la région postérieure. [16] [17]

Cette résorption va abaisser l'os et laisser le muscle plus en contact avec le bord de la prothèse, ce qui favorise sa déstabilisation. Chose qui se manifeste dans notre étude par la plus petite mesure.

Les limites de ce travail découlent de la méthode employée puisque l'étude nécessitait l'intervention après que le praticien ait déjà réalisé l'empreinte secondaire et obtenu le modèle secondaire du patient. La méthode de mise en moufle avec le grattage ultérieur nous a quelquefois donné de légères différences entre les 8 porte empreintes de chaque patient. D'autre part, le silicone de moyenne viscosité étant en rupture au Maroc durant la période de l'étude, nous avons utilisé de l'oxyde de zinc eugénol (l'impression past ${ }^{\circledR}$ ) en retardant l'insertion du PEI en bouche jusqu'à ce que la consistance du matériau permette l'enregistrement du joint. [18] [19] [20]

D'un autre côté, la gêne des patients au début des enregistrements et une légère fatigue à la fin, pourraient avoir un impact sur l'intensité des mouvements effectués et ainsi sur les profondeurs du sillon alvéolo-lingual qui en découlent.

\section{CONCLUSION}

La langue est un organe très dynamique dont les mouvements assurent différentes fonctions orales. Ces mouvements doivent absolument être respectés lors de l'enregistrement de l'empreinte secondaire.

Il ne semble pas qu'un seul mouvement de la langue puisse affecter une région précise de l'arcade mandibulaire édentée indépendamment des autres mouvements. Toute région de l'arcade est influencée par les différents mouvements linguaux.

Il est donc important d'insister sur tous les mouvements pour chaque région de l'arcade mandibulaire lors de la prise de l'empreinte secondaire afin d'obtenir une prothèse totale mandibulaire stable et qui répond à toutes les demandes esthétiques et fonctionnelles. Il serait judicieux de mener d'autres études pour mieux comprendre les mouvements influençant la profondeur du sillon alvéolo-lingual lors de l'enregistrement $\mathrm{du}$ joint lingual. Ces études devraient concerner un échantillon plus large que celui exploité dans la notre pour obtenir des résultats plus significatifs et éviter le maximum de biais.

\section{REMERCIEMENTS}

Nous remercions vivement messieurs les prothésistes KHALID TOUZRI et HASSAN BOURHABA. 


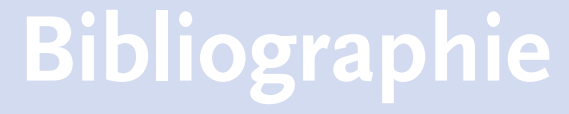

[1] Netter FH. Atlas d'anatomie humaine. Masson édit, Paris, 2009

[2] Schoendorff R, Millet C. Rétention en prothèse complète. EMC Odontologie, 23-325B-05, Elsevier, Paris, 1995.

[3] Gibert Y, Soulet H, Blandin M. Phénomènes rétentifs en prothèse adjointe complète. EMC Odontologie, 23-325-B05, Elsevier, Paris 1987.

[4] Hue O, Berteretche MV. Prothèse complète réalités cliniques, solutions thérapeutiques. Quintessence International, Paris, 2004.

[5] Pompignoli M, Doukhan JY, Raux D. Prothèse complète clinique et laboratoire; tome1. CdP édit, Rueil Malmaison, 2005.

[6] Lejoyeux J. Les empreintes dans le traitement de l'édentation totale. CdP édit, Paris, 1986.
[7] Jaudoin P, Millet C, Mifsud S. Empreinte en prothèse amovible complète. EMC Odontologie: 23-325-C-10, Elsevier, Paris, 2006.

[8] Postaire M. Empreinte secondaire mandibulaire avec joint périphérique aux polyéthers. Réalités Cliniques 1997; 8(4): 383-388.

[9] Berteretche MV. Prothèses et matériaux d'empreintes. Dossiers ADF, 2007, Commission des dispositifs médicaux.

[10] Lejoyeux J. Introduction à la prothèse complète : matériaux et techniques d'empreintes. Maloine édit, Paris, 1964.

[11] Arlsson GE. Clinical morbidity and sequelae of treatment with complete denture. J Prosthet Dent 1998; 79; 17-23.

[12] Jacobson Te, Krol AJ A. Contemporary review of the factors involved in complete denture, retention, stability and support. Part. 1. Retention. J Prosthet Dent 1983; 49: 5-15.

[13] Budtz-Jorgensen E, Clavel R. La prothèse totale : théorie, pratique et aspects médicaux. Masson édit, Paris, 1995.
[14] Jeffcoat M-K. Osteoporosis: a possible modifying factor in oral bone loss. Ann Periodontol 1998; 3: 312-321.

[15] Mercier P. Ridge reconstruction with hydroxylapatite. Part 2. Oral Surg Oral Med Oral Pathol. 1998; 65: 505-10.

[16] Schreinemakers J. La logique en prothèse complète. G.J. \& D. Tholen N.v édit, Utrecht Pays-Bas, 1968.

[17] Begin M, Rohr M. Le joint vélo-palatin en prothèse complète maxillaire. Cah Proth 1984; 43: 55-78.

[18] Lacoste-Ferre MH, Dandurand J, Blandin M, Pomar P, Quels élastomères pour quelles empreintes? Cah. Proth 2006; 136: 51-58.

[19] Lacroix P, Laurent M, Margossian P, Laborde G. Quels sont les critères de choix des porte-empreintes et adhésifs ? Strat. Proth 2004; 4(5): 337-342.

[20] Van Noort R. Introduction to dental materials, Second edition, Mosby édit. Edinburgh, 2002. 